\title{
PID and Fuzzy-PID Control Model for Quadcopter Attitude with Disturbance Parameter
}

\author{
E. Kuantama, T. Vesselenyi, S. Dzitac, R. Tarca
}

\author{
Endrowednes Kuantama*, Tiberiu Vesselenyi, \\ Simona Dzitac, Radu Tarca \\ University of Oradea \\ 1 Universitatii St., Oradea, Romania \\ endrowednes@gmail.com,vtiberiu@uoradea.ro, \\ simona@dzitac.ro,rtarca@uoradea.ro \\ *Corresponding author: endrowednes@gmail.com
}

\begin{abstract}
This paper aims to present data analysis of quadcopter dynamic attitude on a circular trajectory, specifically by comparing the modeling results of conventional Proportional Integral Derivative (PID) and Fuzzy-PID controllers. Simulations of attitude stability with both control systems were done using Simulink toolbox from Matlab so the identification of each control system is clearly seen. Each control system algorithm related to roll and pitch angles which affects the horizontal movement on a circular trajectory is explained in detail. The outcome of each tuning variable of both control systems on the output movement is observable while the error magnitude can be compared with the reference angles. To obtain a deeper analysis, wind disturbance on each axis was added to the model, thus differences between each control system are more recognizable. According to simulation results, the Fuzzy-PID controller has relatively smaller errors than the PID controller and has a better capability to reject disturbances. The scaling factors of gain values of the two controllers also play a vital role in their design.

Keywords: Quadcopter, PID, fuzzy-PID, Simulink, attitude.
\end{abstract}

\section{Introduction}

A quadcopter, as its name suggests, has four symmetrical rotor-propellers which are mounted at the same height on each edge, forming a cross with identical radius from its center [9]. Each rotor propeller creates an upward thrust which is highly maneuverable by controlling the speed variation, thus allowing the quadcopter for vertical take-off, landing and even hovering. However, to achieve good stability as well as to minimize interference in every movement, the quadcopter attitude must be converted into a suitable control algorithm. This is considered to be a challenging problem because quadcopters have a complex dynamic system with high nonlinearities, strong couplings, and under actuation [13]. According to the control strategy of quadcopters, the flight control systems can be divided into 3 types, i.e., linear flight control system, nonlinear flight control system, and learning based flight control system [5]. The linear flight control system, or feedback linearization, is a very useful method to decouple and linearize quadcopter attitude models. This paper will review in detail the algorithm of linear control system for quadcopters using conventional PID (Proportional Integral Derivative) and Fuzzy-PID control. Considering the advantages of the Ziegler-Nichols rules for tuning the PID controller [22] and the feedback linearization method, we adopted this algorithm for the quadcopter attitude control and we analyzed the obtained results. The way of tuning PID controller parameters with mathematical modeling into feedback linearization for stability attitude is crucial. The way of tuning PID controller parameters with mathematical modeling using feedback linearization is crucial for attitude stability. On the other hand, Fuzzy-PID algorithms have limitless ranges to flawlessly perform when rapid changes are encountered in high dynamic environments, moreover 
it does not require complex mathematical models [7]. A comparison of the PID and Fuzzy-PID tuning is presented in which both controllers have been studied based on a dynamic model of a quadcopter.

Modeling of the quadcopter control and movement has been done using Simulink Matlab. Through this modeling and control algorithm, differences between angular velocity with and without PID and FUzzy-PID control can be studied, as well as the impact of disturbances on the control system. To understand the control system of quadcopters, firstly one must comprehend the concept of quadcopter attitude based on the Newton-Euler equation. Details of the quadcopter dynamic movement using this equation have been already covered in previous researches [12], [15]. This time, Newton-Euler equation is adapted to develop a quadcopter attitude model in which the quadcopter moves in a full circle while using PID and Fuzzy-PID control systems for dynamic movement control. Another aim of this study is to investigate the behavior of the quadcopter control system when faced with wind disturbances from multiple directions.

\section{Quadcopter attitude modelling}

Vertical thrust is the result of combined angular velocity of four rotors which are directly connected to the propellers, thus driving the quadcopter attitude as a whole. Naturally, rotor propellers placed in opposed position will rotate in the same direction and side-by-side ones will rotate oppositely. Controlling the angular velocity of the four rotors will consequently result in a dynamic movement with force and moment transmission which can lead to lift, pitch, roll or yaw rotations moment inertia. Fig. 1. illustrates these rotations, and are explained as follows: rotation around $\mathrm{X}$-axis as roll movement, rotation around $\mathrm{Y}$-axis as pitch movement, and rotation around Z-axis as yaw movement.
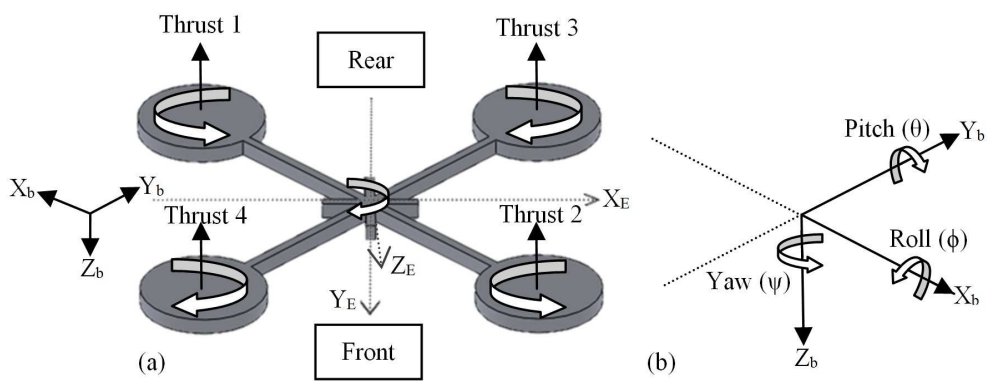

Figure 1: Quadcopter schematic: (a) The structure of a quadcopter (b) Description of Pitch, Roll, and Yaw angles

Quadcopter attitude has a total of six Degrees of Freedom (DOF), half are allotted to translational position in an Earth inertial frame and the rest to angular positions of the quadcopter's rigid body. All DOFs are covered by the linear and angular orientation vectors of the quadcopter, thus it can be seen that the quadcopter has four inputs from the rotor propellers and six state outputs of the dynamic and complex system. In order to control these, the process of quadcopter modeling can be approached by interpreting any attitude movement with two reference frames in the form of the Earth inertial frame (coordinate system) axes $\left(X_{E}, Y_{E}, Z_{E}\right)$ and the body frame axes $\left(X_{b}, Y_{b}, Z_{b}\right)$. From this point, we can formulate the equations and parameters needed to establish the transformation matrix based on the Newton-Euler equation model [3]. Each parameter needed for attitude modeling can be seen in Table. 1. 
Table 1: Parameter for attitude modelling algorithm

\begin{tabular}{cccccc}
\hline Movement & $\begin{array}{c}\text { Translation } \\
\text { Positions }\end{array}$ & $\begin{array}{c}\text { Angular } \\
\text { Positions }\end{array}$ & $\begin{array}{c}\text { Translation } \\
\text { Velocity }\end{array}$ & $\begin{array}{c}\text { Angular } \\
\text { Velocity }\end{array}$ & $\begin{array}{c}\text { Inertial } \\
\text { Matrix }\end{array}$ \\
\hline Roll & $\mathrm{x}$ & $\phi$ & $v_{\mathrm{x}}$ & $\mathrm{p}$ & $\mathrm{I}_{\mathrm{xx}}$ \\
Pitch & $\mathrm{y}$ & $\theta$ & $v_{\mathrm{y}}$ & $\mathrm{q}$ & $\mathrm{I}_{\mathrm{yy}}$ \\
Yaw & $\mathrm{z}$ & $\psi$ & $v_{\mathrm{z}}$ & $\mathrm{r}$ & $\mathrm{I}_{\mathrm{zz}}$ \\
\hline
\end{tabular}

\subsection{Newton-Euler kinematics model}

The Newton-Euler modeling was adapted in designing linear $\left(L_{T}\right)$ and angular motion $\left(R_{T}\right)$ expressions of the quadcopter which can deliver lift up forces where change of attitude occur on the orientation of the body frame axes with respect to the Earth inertial frame axes. Orientation change of body frame axes results in angle alteration on roll $-R_{x}(\phi)$, pitch $-R_{y}(\theta)$, and yaw $-R_{z}(\Psi)$, respectively. Based on the kinematic moving frame theory, the equations using transformation matrices for angular motion on each axis or for every movement can be written. Matrix transformation between two rectangular coordinate systems is orthogonal and can be converted to $3 \times 3$ matrices for every axis. Eq.(1). shows the rotation matrix around X-, Y-, and $\mathrm{Z}$-axis and the transformation of the overall rotation movement $\left(R_{T}\right)$ of the body frame can be calculated using Eq.(2).

$$
\begin{gathered}
R_{x}(\phi)=\left[\begin{array}{ccc}
1 & 0 & 0 \\
0 & \cos \phi & \sin \phi \\
0 & -\sin \phi & \cos \phi
\end{array}\right] ; R_{y}(\theta)=\left[\begin{array}{ccc}
\cos \theta & 0 & -\sin \theta \\
0 & 1 & 0 \\
\sin \theta & 0 & \cos \theta
\end{array}\right] ; R_{z}(\psi)=\left[\begin{array}{ccc}
\cos \psi & \sin \psi & 0 \\
-\sin \psi & \cos \psi & 0 \\
0 & 0 & 1
\end{array}\right] \\
R_{T}=R_{x}(\phi) R_{y}(\theta) R_{z}(\psi)
\end{gathered}
$$

The rate of change in linear motion from one position to another is related to the angular velocity and the difference in angle which will result in alteration of system output $\mathrm{x}, \mathrm{y}, \mathrm{z}$ position. This alteration can be computed using the angular rates measured by sensor of the quadcopter (p, q, r). The rotation angle in Eq.(1). is used to compute the angular velocity of the quadcopter. The angular velocity with respect to the body frame can be computed by transforming the matrix from angular position $\left[\begin{array}{lll}p^{\prime} & q^{\prime} & r \prime\end{array}\right]^{T}$ to Euler angle rates $\left[\begin{array}{lll}\phi^{\prime} & \theta \prime & \psi^{\prime}\end{array}\right]^{T}$ given in Eq.(4). In fact, the values of $\mathrm{p}, \mathrm{q}$ and $\mathrm{r}$ are obtained through orientation sensor readings on the quadcopter, thus the alteration of the angles of the body frame can be computed using the inverse of Eq.(4)., as it is seen in Eq.(5).

$$
\begin{gathered}
\mathcal{V}=\left[\begin{array}{l}
p \\
q \\
r
\end{array}\right]=R_{x}(\phi) R_{y}(\theta) R_{z}(\psi)\left[\begin{array}{c}
0 \\
0 \\
\psi \prime
\end{array}\right]+R_{x}(\phi) R_{y}(\theta)\left[\begin{array}{c}
0 \\
\theta \prime \\
0
\end{array}\right]+R_{x}(\phi)\left[\begin{array}{c}
\phi \prime \\
0 \\
0
\end{array}\right] \\
{\left[\begin{array}{l}
p \\
q \\
r
\end{array}\right]=\left[\begin{array}{ccc}
1 & 0 & -\sin \theta \\
0 & \cos \phi & \sin \phi \cos \theta \\
0 & -\sin \phi & \cos \theta \cos \phi
\end{array}\right]\left[\begin{array}{l}
\phi \prime \\
\theta \prime \\
\psi \prime
\end{array}\right]} \\
{\left[\begin{array}{l}
\phi \prime \\
\theta \prime \\
\psi \prime
\end{array}\right]=\left[\begin{array}{ccc}
1 & \sin \phi \tan \theta & \cos \phi \tan \theta \\
0 & \cos \phi & -\sin \phi \\
0 & \sin \phi / \cos \theta & \cos \phi / \cos \theta
\end{array}\right]\left[\begin{array}{l}
p \\
q \\
r
\end{array}\right]}
\end{gathered}
$$

The dynamic movement of the quadcopter, both on angular and translational basis are expressed with the Newton-Euler equation of motion as shown in Eq.(6). The angular acceleration 
of the inertia $(I \omega \prime)$, the centripetal forces $(\omega \times(I \omega))$ and the gyroscopic forces $(\gamma)$ are equal to the external torque applied to the bodyframe $(\tau)$. From this equation, angular velocity vector $(\omega \prime)$ can be determined as shown in Eq.(8).

$$
\begin{gathered}
\tau=I \omega \prime+(\omega \times(I \omega))+\gamma \\
\omega \prime=I^{-1}[-\omega \times(I \omega)+\tau-\gamma] \\
{\left[\begin{array}{c}
p \prime \\
q^{\prime} \\
r \prime
\end{array}\right]=I^{-1}\left(\left[\begin{array}{l}
\left(I_{y y}-I_{z z}\right) q r \\
\left(I_{z z}-I_{x x}\right) p r \\
\left(I_{x x}-I_{y y}\right) p q
\end{array}\right]+\left[\begin{array}{c}
\tau_{\phi} \\
\tau_{\theta} \\
\tau_{\psi}
\end{array}\right]-I_{m} \omega_{i}\left[\begin{array}{c}
q \\
-p \\
0
\end{array}\right]\right)}
\end{gathered}
$$

Where $I_{m}$ is the Inertia matrix, $\omega$ is the angular velocity, $\omega /$ is the angular position, and $\omega_{i}$ is the scalar angular speed of rotor. The inertia matrix for the quadcopter is diagonaldue to the symmetry of the quadcopter. Quadcopter's mass and its geometric distribution (especially inertia) are affecting the entire dynamics of the system. The symbols $I_{x x}, I_{y y}, I_{z z}$ are the moments of inertia matrices which are the torque of the rotors.

\subsection{Circular trajectory model}

Analysis of attitude control was done by modeling the dynamic of the quadcopter moving on a circular trajectory. This implies that the X-Y position on the circular trajectory needs to be computed using stability control on roll and pitch motions while yaw angle is zero. The path of motion along $\mathrm{X}$ - and $\mathrm{Y}$-axes can be seen in Fig. 2. [18].

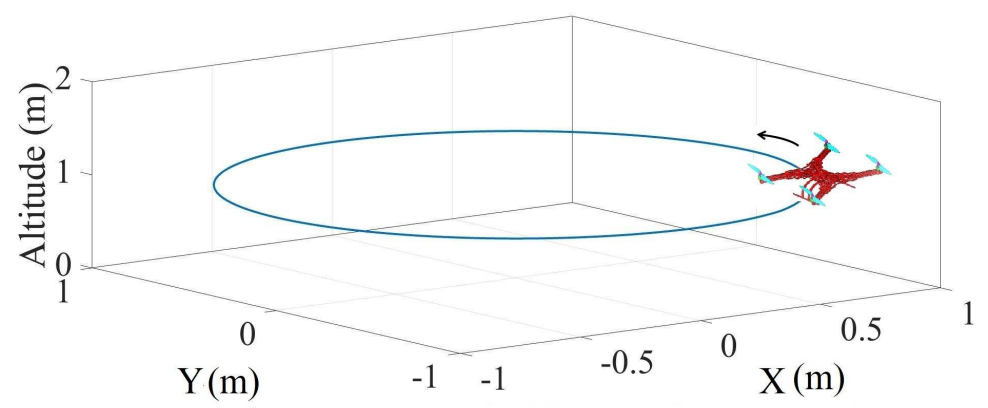

Figure 2: Quadcopter attitude trajectory model

As it is known, two rotor propellers will cause movement on each axis around the center of gravity. In this modeling, rotors placed on the same axis will generate opposite thrust values, or in other words, the increasing thrust on one side is linear with the decreasing thrust on the other side in the same axis. The rotation torques on the body frame which derives from the rotor on each axis are dependent upon two parameters, i.e., the distance between rotor and quadcopter's centroid (L) and the magnitude of thrust generated by the rotors on the same axis (X-axis or Y-axis). The torque equation on the body frame on each axis is given in Eq.(9).

$$
\tau_{x}=L\left(T_{\text {rotor } 4}-T_{\text {rotor } 2}\right) ; \tau_{y}=L\left(T_{\text {rotor } 3}-T_{\text {rotor } 1}\right)
$$

From the quadcopter theory and attitude parameter, basic modeling was done with only a proportional control system. Close loop feedback was designed to ensure a good functionality of the algorithm for quadcopter attitude, as well as to evaluate the output of the angular velocity on every axis. There are three subsystems of the designed model, i.e., input parameters in form 
of inertia matrices $I_{x x}, I_{y y}, I_{z z}$ and distance between rotor and centroid (L), the algorithm for quadcopter attitude and circular trajectory, and lastly the output in form of quadcopter movement on $\mathrm{X}$ - and $\mathrm{Y}$-axis. The simulation block diagram for this model can be seen in Fig. 3. The quadcopter parameters used in this model are as follows: $\mathrm{L}=0.25 \mathrm{~m}$ and $I_{x x}=I_{y y}=0.005$ $k g \cdot \mathrm{m}^{2}$. Because the modeling focused only on X-and Y-axis, $I_{z z}$ was temporarily unneeded.

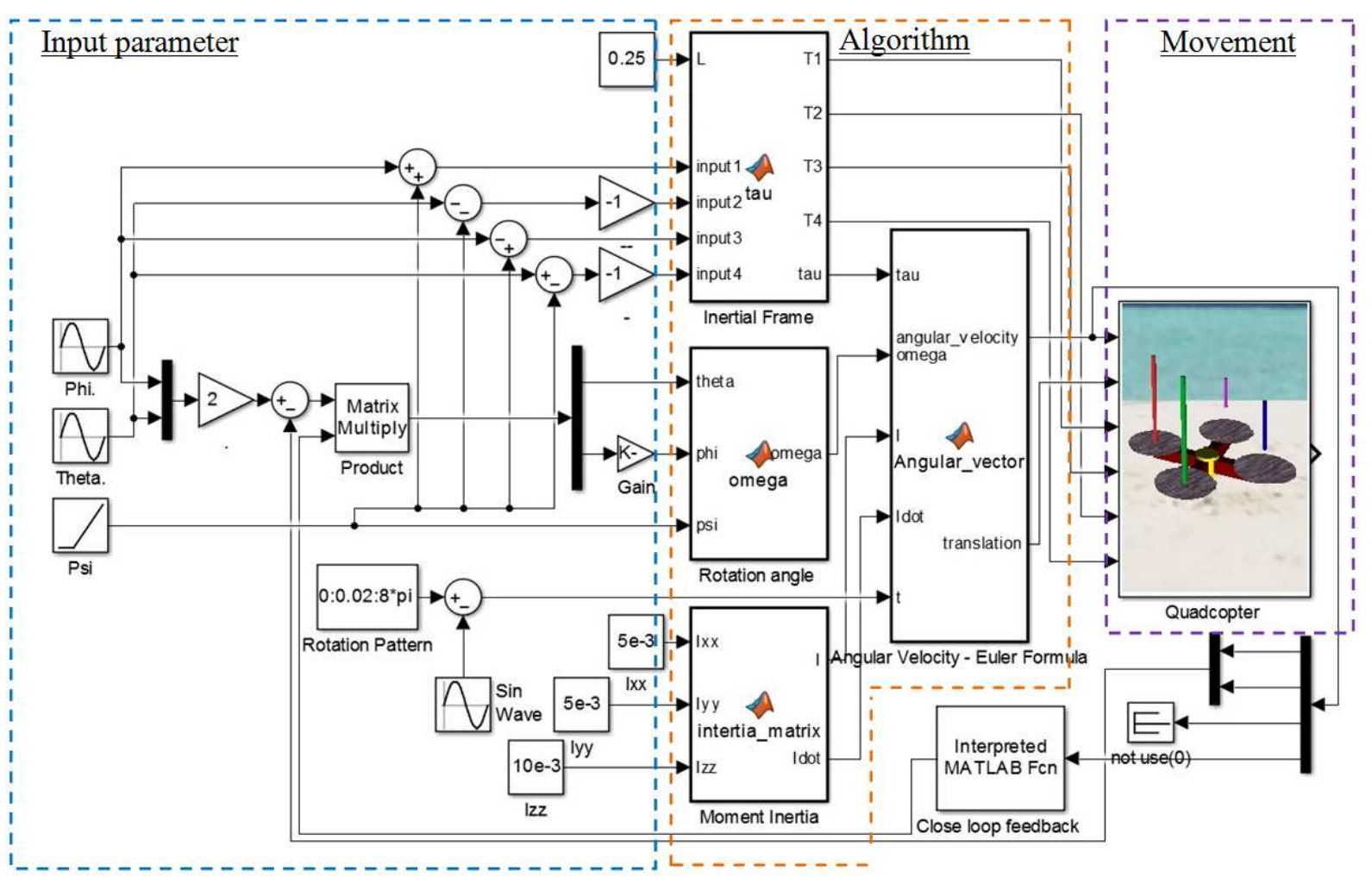

Figure 3: Simulation block diagram

\section{Controller architecture}

Analysis of the matrices function for every attitude reveals the fact that the quadcopter model requires a close feedback linearization control system for balancing and localization. This control architecture is necessary to enhance the dynamic control response of the quadcopter with respect to the quadcopter body frame. In this study, the movement on a circular trajectory was investigated and to achieve stability, PID and Fuzzy-PID control systems have been used [2]. Generally, the control system for the quadcopter moving on a circular trajectory, in fly/hover mode, or in other attitude movement can be done with the same control algorithm to attain stability. Fig. 4 shows the quadcopter control system with close loop feedback and linearized PID or Fuzzy-PID control. In this paper, the controller for X and Y position will compute the desired roll and pitch angles depending on the desired values for quadcopter movement on each axis. These angles are fed into the "Attitude" block and with a closed-loop feedback the controller can continuously do a linearization process against any possible error factor. 


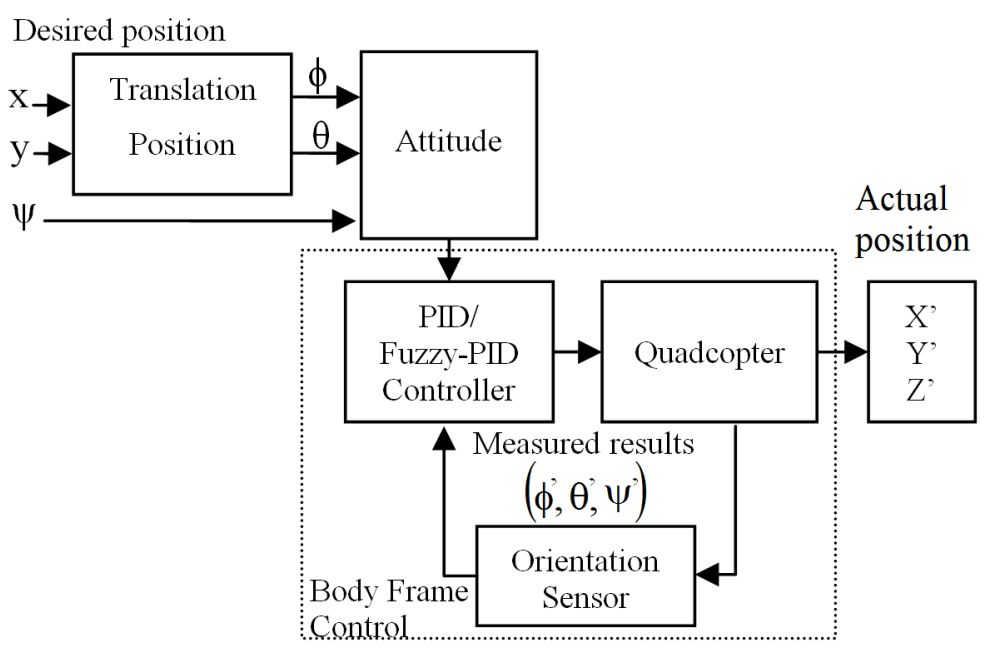

Figure 4: Quadcopter control system diagram

\subsection{PID control parameter}

Implementation of PID controller tuned by Ziegler-Nichols rules to the linear model of quadcopter can be done by adopting the parameter feedback linearization items which are acting on the quadcopter position. The advantages of the PID controller is its simple control structure and the ease of implementation [4]. A PID controller regards an "error" value as the difference between a measured variable and a desired set-point. The equation of a PID controller is:

$$
\begin{gathered}
U(t)=K_{p} e(t)+K_{i} \int_{0}^{t} e(\tau) d \tau+K_{d} \frac{d}{d t} e(t) \\
e(t)=X_{d}(t)-X(t)
\end{gathered}
$$

In which $\mathrm{U}(\mathrm{t})$ is the control output, $\mathrm{e}(\mathrm{t})$ is the difference between the desired stated $X_{d}(t)$ and the actual state $\mathrm{X}(\mathrm{t})$, and $K_{p}, K_{i}, K_{d}$ are the proportional, integral, and derivative gains, respectively. Alteration in pitch and roll angles may occur based on the torque generated torque by the rotors on $\mathrm{X}$-axis or $\mathrm{Y}$-axis on the body frame. In this modeling, it was sufficient just to manage the $K_{p}$ and $K_{d}$ control parameters ( $\mathrm{P}$ and D control), as $K_{i}$ was used to reduce the final error of the system and thus it is not needed [10]. The nominal coefficients of PD used to obtain the robust stability equation are based on the angular velocity equation of the quadcopter [14]. Hence, the PD controller for the quadcopter in horizontal position can be calculated using Eq.(12). based on two relations, i.e., the torque generated by each horizontal axis (shown in Eq.(9).) and also the equation of angle alteration (shown in Eq.(4).). The total mass of the quadcopter will have impact on the generated thrust, whereas the torque will be influenced by the moments of inertia (I).

$$
\tau_{x, y}=\left[\begin{array}{c}
L\left(T_{\text {rotor } 4}-T_{\text {rotor } 2}\right) \\
L\left(T_{\text {rotor } 3}-T_{\text {rotor } 1}\right]
\end{array}\right]=\left[\begin{array}{c}
\left(K_{\phi, p}\left(\phi_{p^{\prime}}-\phi \prime\right)+K_{\phi, D}\left(\phi_{d^{\prime}}-\phi \prime\right)\right) I_{x x} \\
\left(K_{\theta, p}\left(\theta_{p^{\prime}}-\theta \prime\right)+K_{\theta, D}\left(\theta_{d^{\prime}}-\theta \prime\right)\right) I_{y y}
\end{array}\right]
$$

The performance of PD controller was tested using stability simulation of quadcopter during the movement on the circular trajectory. The PD controller parameters can be seen in Fig. 5. where the values of parameters $K_{p}$ and $K_{d}$ were obtained based on manual tuning which was done to get magnitude stability of the attitude value. Parameter setting principles are based on the Ziegler-Nichols PD. The amount of error that occurs on the roll angle is not significant, so 
it only takes the proportional gain value to adjust the generated output variable. The purpose of the stabilization is to obtain the same output of angular velocity in any desired position.

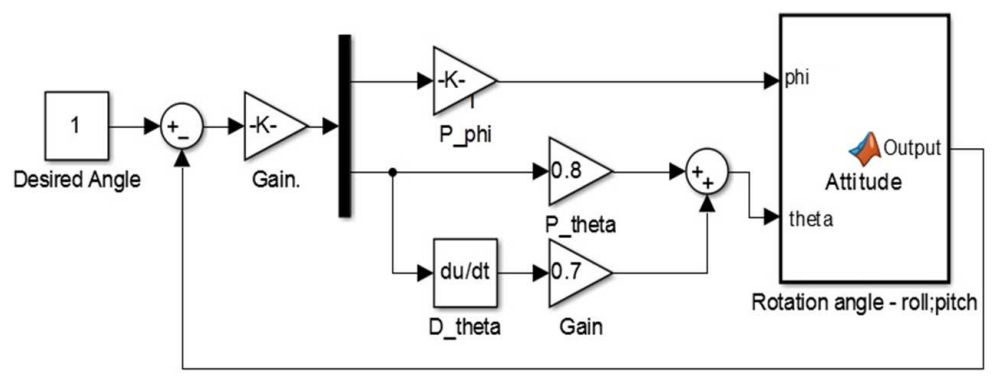

Figure 5: Quadcopter PID control system Simulink block diagram

\section{$3.2 \quad$ Fuzzy-PID control parameter}

As for the combination between PID control theory and Fuzzy control theory, the FuzzyPID control aims to establish a two variable continuous function between the PID parameters, absolute error value and absolute error value changes [11]. It has a self-tuning characteristic to automatically tune the control parameter to obtain optimal output [21]. Optimal output can be obtained by adjusting the gain values of $K_{p-F u z z y}, K_{i-F u z z y}, K_{d-F u z z y}$ based on the comparison of the input in form of error and error rate values. The input will be analyzed using a Fuzzy system in which if-then rules are applied as tuning parameter set of the received input [1], [19]. In accordance with the typical PID controller, the gain values of the Fuzzy-PID controller can be adjusted with the parameters as seen on Eq.(13). [8].

$$
U_{F u z z y-P I D}=K_{p-F u z z y} e(t)+K_{i-F u z z y} \int_{0}^{t} e(\tau) d \tau+K_{d-F u z z y} \frac{d}{d t} e(t)
$$

A Fuzzy-PID controller is proposed in this work to control the quadcopter stability in terms of attitude position on circular trajectory, considering that the quadcopter movements are heavily influenced by non-linear factors and dynamic coupling. The Fuzzy-PID control attitude on pitch and roll positions are designed separately in order to accurately control the PID gain values. As it is known through PID control parameter, in this design, the PD gain values are enough to control the quadcopter movement on a horizontal path.

The position of the quadcopter on a circular trajectory depends on the acceleration of the quadcopter and is based on the change in pitch and roll angles. Each angle changes will be monitored and controlled using a Fuzzy-PID controller. Each controller has 2 membership functions where the first one, represented by In1, receives the current angle which is obtained from the orientation sensor reading of the quadcopter attitude; The other one (In2) reseives the error rate which is obtained from comparing current angle value with the reference angle value. Through these 2 membership functions, the Fuzzy PID system with If-Then logic or Fuzzy rule parameters can be applied to reduce the position error value on output in the form of quadcopter movement. Mamdani-type inference is used for the inference engine with centroid defuzzification method [16] and it lays out the foundation rules for the Fuzzy system [20]. Each input and output are divided into 7 groups of linguistic variables, i.e., negative large (NL), negative medium $(\mathrm{NM})$, negative small (NS), zero (Z), positive small (PS), positive medium (PM), and positive large (PL). These linguistic variables express the degree of error and also the error rate in roll and pitch angle while moving on the circular trajectory. The details of these variables can be seen in Fig. 6. Two inputs and one output are normalized in the same interval $[-1,1]$ with a 


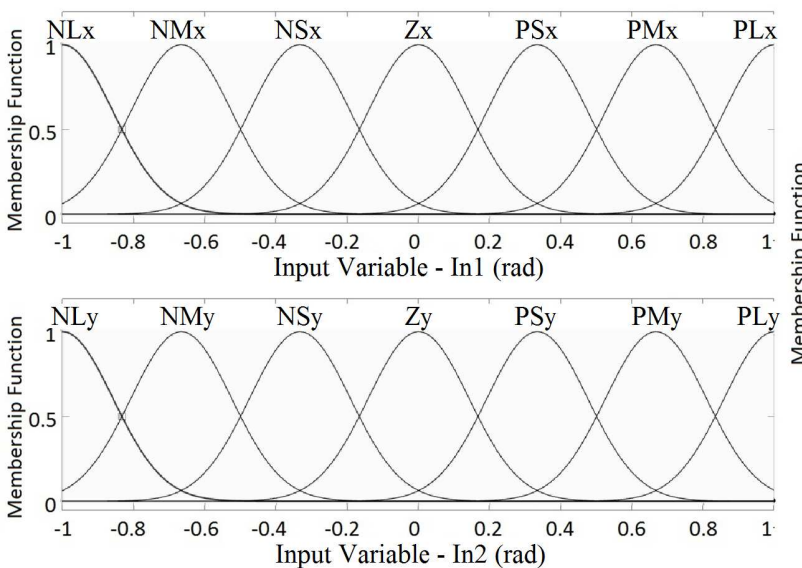

(a)

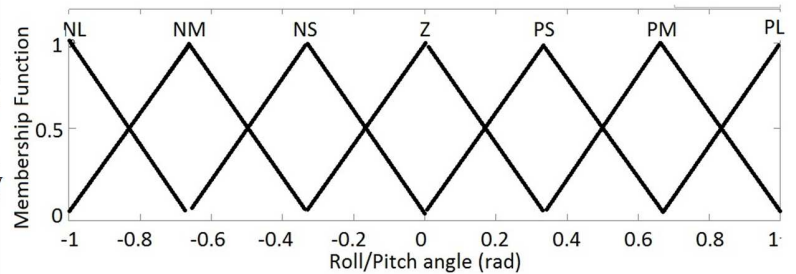

(b)

Figure 6: The membership function of the Fuzzy logic controller: (a) Input (b) Output

scaling factor variable of 0.2 for every linguistic group. The interval value must be equivalent with the circular trajectory movement values in the designed roll and pitch angle so that each scale will cover detailed changes. Table 2 shows 49 fuzzy inference rules of this control action with the possible combinations of the two input values.

Table 2: Fuzzy inference rules for quadcopter

\begin{tabular}{|c|c|c|c|c|c|c|c|}
\hline In1/In2 & NLy & NMy & NSy & Zy & PSy & PMy & PLy \\
\hline NLx & NL & NL & NL & NL & NM & NS & Z \\
\hline NMx & NL & NL & NL & NM & NS & Z & PS \\
\hline NSx & NL & NL & NM & NS & Z & PS & PM \\
\hline Zx & NL & NM & NS & Z & PS & PM & PL \\
\hline PSx & NM & NS & Z & PS & PM & PL & PM \\
\hline PMx & NS & Z & PS & PM & PL & PL & PL \\
\hline PLx & Z & PS & PM & PL & PL & PL & PL \\
\hline
\end{tabular}

$\operatorname{In} 1=$ derivative value/error position; $\operatorname{In} 2=$ error rate

After establishing the fuzzy parameter values and its rules, the Fuzzy-PID system as seen in Fig. 7 can be designed. Two identical Fuzzy logic controllers are designed to control the movement in roll and pitch angles on the circular trajectory. Inputs received by the Fuzzy system are measured through the error normalization factor (GE) and the change in measurement normalization factor (GCE). This factor can be calculated based on the values of $K_{p-F u z z y}(K p f)$, $K_{i-F u z z y}(K i f), K_{d-F u z z y}(K d f)$, and the allowed maximum error as seen on Eq.(14). and Eq.(15).

$$
\begin{gathered}
G E=\frac{1}{\text { max.error }} \\
G C E=G E *\left(K_{p f}-\sqrt{K_{p f}^{2}-4 K_{i f} K_{d f}} \frac{K_{i f}}{2}\right)
\end{gathered}
$$

Output responses of the Fuzzy are measured through response de-normalization factor (GU) and change in the response de-normalization factor (GCU) as seen in Eq.(16) and Eq.(17). This scaling factor is depending on gain information of conventional PID.

$$
G U=\frac{K_{d f}}{G C E}
$$




$$
G C U=\frac{K_{i f}}{G E}
$$

In this design, output normalization was done by directly triggering the changes of proportional and derivative action in which only the GU variable is used. The amount of designed error normalization factor is 0.5 with a change in measurement normalization factor of 0.3 . The output of the PID controller will be used in Euler integration for the quadcopter attitude on a circular trajectory.

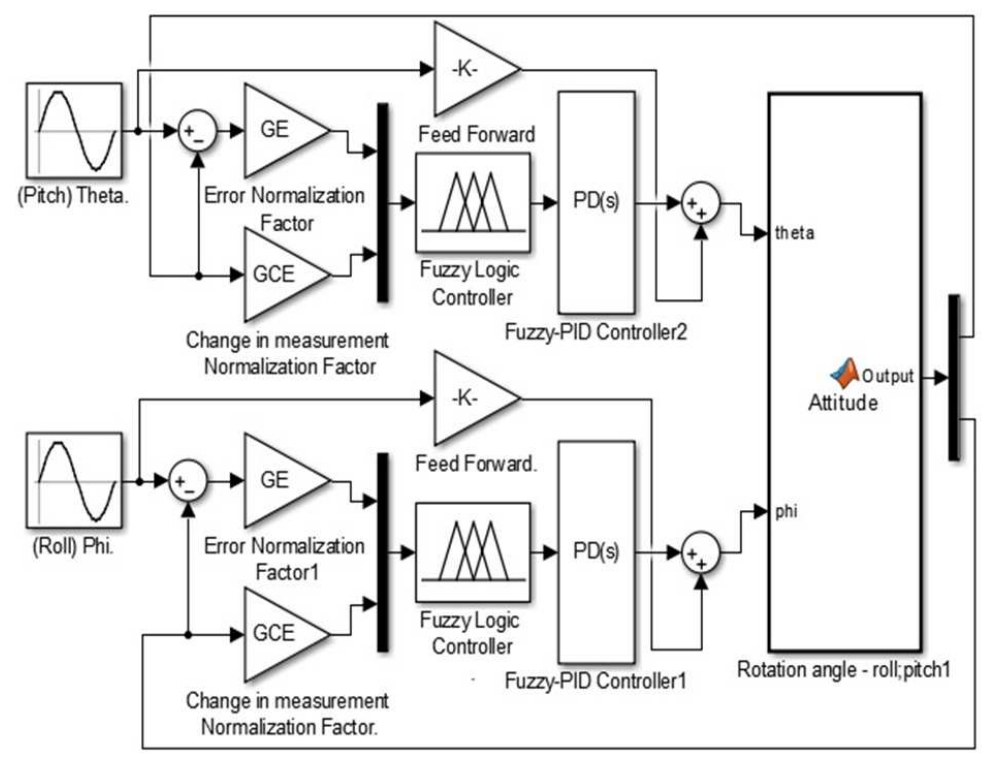

Figure 7: Quadcopter Fuzzy-PID control system Simulink block diagram

\section{Simulation results}

The circular movement of the quadcopter was simulated using Simulink modeling which demonstrated the applicability of designed control in terms of control system stability on roll and pitch axes with steady high performance. The results are represented as angular velocity and position control responses of the control algorithm. Circular trajectory modeling was performed by adjusting pitch and roll angle in horizontal position as shown in Fig. 8(a). which was also used as reference angle. In quadcopter movement, the angular velocity algorithm can be developed using Eq.(9). and the modeling was done with a closed loop system. Practically, output angles for roll and pitch were obtained from attitude reading or orientation sensor data and the outcome was compared with the reference angles to observe how much error occurred. Fig. 8(b). shows the output roll and pitch angles, and the differences between the desired angles and the outputs.

Due to the symmetry of the quadcopter, the same attitude error occurred on pitch and roll angles where noticeable error was seen in the settling time of 3-4 seconds and also in the settling time 7-8 seconds with an overshoot greater than $20 \%$. The differences between the generated attitude and the reference angles proved that the control algorithm is crucial to lessen the error. PID and Fuzzy-PID control systems are essential to cope with this problem and the differences between their usages are seen clearly from the output. The tuning of the PID control parameters for both roll angle and pitch angle loops can be accomplished by adjusting the proportional and derivative coefficient. The proportional gain coefficient is used to manage acceleration and stability, while the integral coefficient is used to reduce permanent fault, and lastly the derivative 


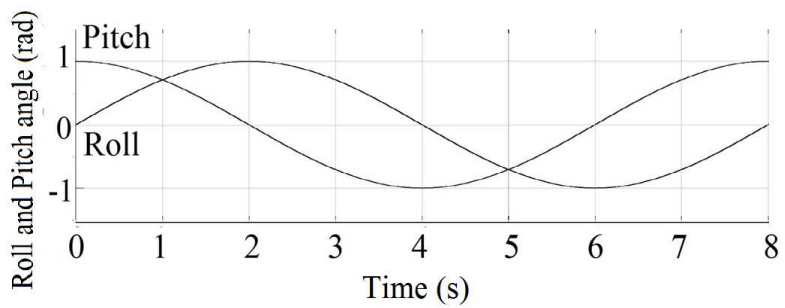

(a) Desired angle

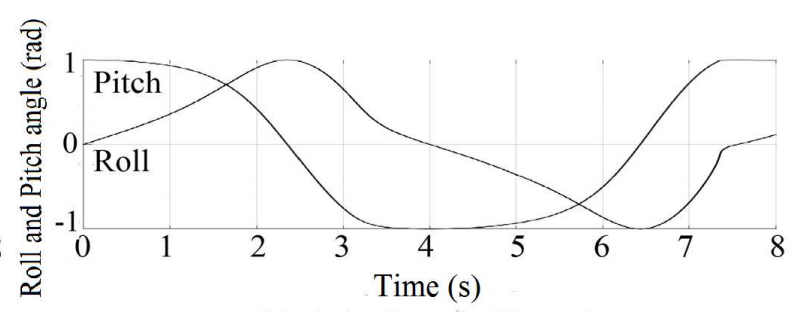

(b) Attitude angle (Output)

Figure 8: Quadcopter roll and pitch angle

coefficient is used to reduce oscillation range and increase stability [17]. No steady state error was observed on roll and pitch angles, thus the PID controller could be simplified into a PD controller with integral gain $K_{i}=0$. The complete position and attitude controller was developed to follow a circular trajectory with values $K_{p}$ of 0.8 and $K_{d}$ of 0.7 , for which the Simulink block diagram is presented in Fig. 5. These gains of the PD controller were used as a set of operation points for the roll and pitch control. Fig. 9. shows the comparison between the performance of the system with PD controller and the reference angle. The variable position output is explained on each axis in which $\mathrm{x}$-axis position is called roll position and $\mathrm{y}$-axis position is called pitch position.

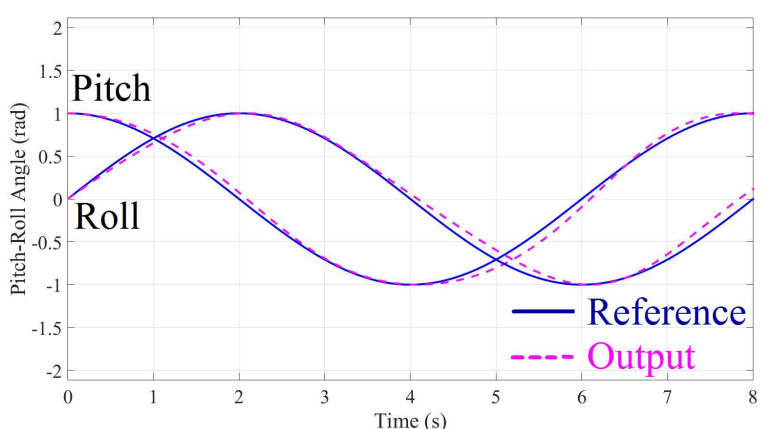

(a)

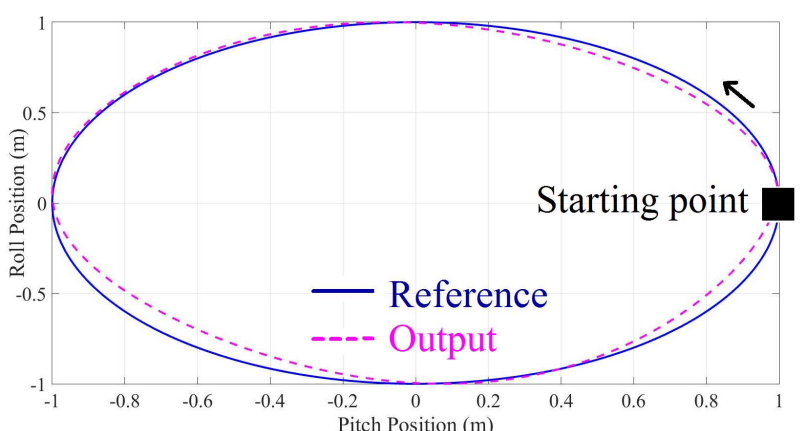

(b)

Figure 9: Quadcopter Attitude with PD control system. (a) Pitch and roll angle (b)Trajectory position

The influence of using PD control is very clear where overall output is almost identical to the reference, establishing an proper movement on circular trajectory. This data attest that the final values of proportional and derivative gain control can maintain proper operation on pitch and roll angles with maximum overshoot value no more than $7 \%$ which occurs on the third quadrant, between 4 to 6 seconds of settling time. This circumstance is producing a shift in position on the trajectory as large as $0.12 \mathrm{~m}$.

The system using the PD control on roll and pitch axis has achieved a satisfying response in circular movement, and then PD control system was replaced with the Fuzzy-PID control system to study the difference. A Fuzzy-PID controller structure with feed forward loop was designed to obtain stabilization at steady state. The Fuzzy rules for pitch and roll angles are alike and the values of Fuzzy-PID sets used for the controllers are also identical. These values can be determined by an adaptive trial and error method. The values of Fuzzy sets for $K_{p f}$ and $K_{d f}$ are 0.288 and 0.33 respectively.Fig. 10. shows the modeling result of horizontal balancing control system on the pitch and roll axis. In the settling time between 0 to 8 seconds, the horizontal balancing control system on the roll axis can maintain the position/angle as well as on the yaw axis. This result shows that the Fuzzy-PID control system is better than the PID control system 


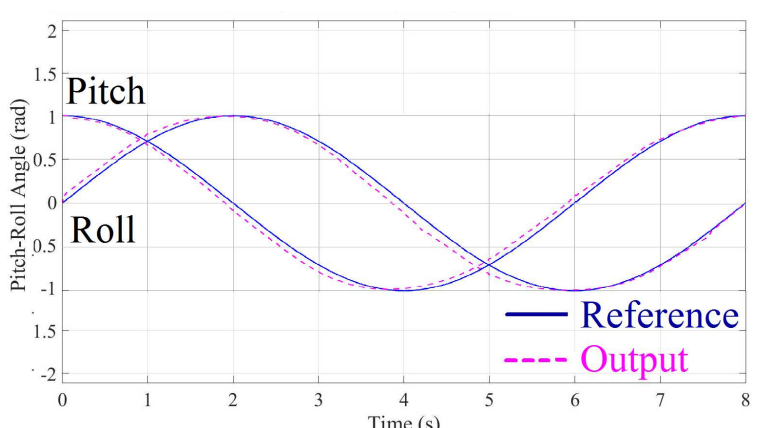

(a)

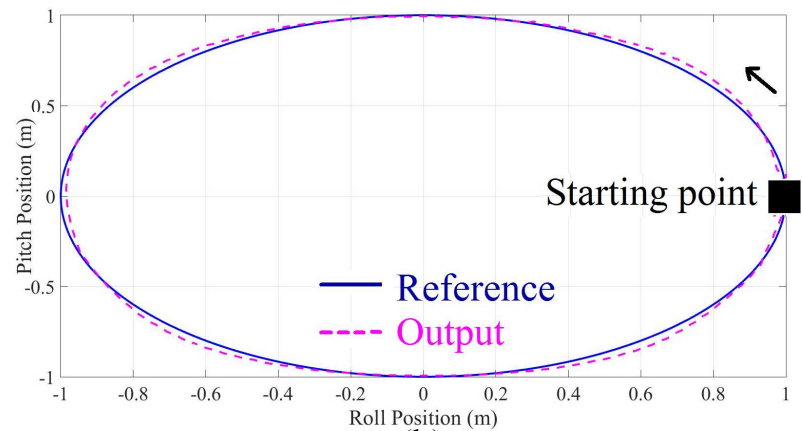

(b)

Figure 10: Quadcopter attitude with Fuzzy-PID control system. (a) Pitch and roll angle (b) Trajectory position

with a position shift less than $0.05 \mathrm{~m}$ and good flight stability on circular movement. This means that the fuzzy logic system can be used to change the output position to the different moments of control coefficients which can be done in the presence of fuzzy control along with the PID control [6]. It can be concluded that both systems are usable in this system and the difference between PID and Fuzzy-PID controller only causing a small error in a no-disturbance condition, allowing the quadcopter to move on the desired circular trajectory.

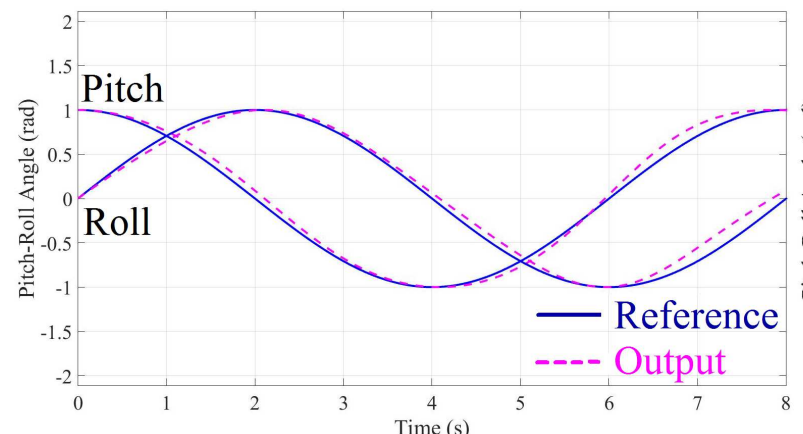

(a)

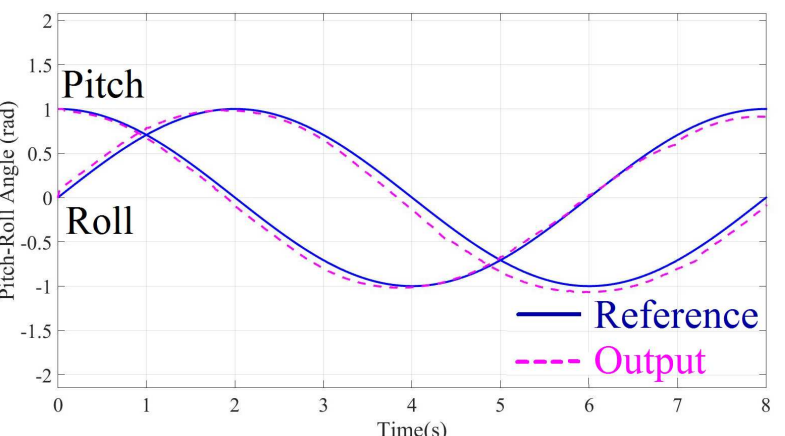

(b)

Figure 11: Attitude control system with disturbance parameter. (a) PID (b) Fuzzy-PID

To find out more about the difference between the two control systems on the quadcopter, a disturbance parameter of wind with $2 \mathrm{~m} / \mathrm{s}$ velocity was added with various combination of wind direction. Discrete gust wind models were used with gain values of $3.5,3.5,3.5 \mathrm{~m} / \mathrm{s}$ and gust wind lengths of 120,120, $80 \mathrm{~m}$ on each axis. Gust wind length is the rise time needed to build up gust wind which expressed with length in each axis. The result of changes in pitch and roll angles with wind directions on each axis can be seen in Fig. 11. Disturbances affect the angle alteration in pitch and roll movement, thus resulting in greater tilt angle of the quadcopter. For a deeper analysis, Fig. 12. shows the detail of changes in quadcopter flight position with various wind directions. During the circular movement with wind effect in $\mathrm{x}$ - and $\mathrm{y}$ - directions, each control system has different behavior. In case of the PID control system, the shift in pitch and roll angles of movement happens almost at every settling time, but significant error in position occurs after 5 seconds of settling time. Overall the fourth quadrant witnesses the maximum angle alteration of 0.14 radians from the reference angle and a maximum position shift of $0.1 \mathrm{~m}$. In the case of the Fuzzy-PID control, it can be clearly seen that the angle alteration happens in each position. This results in a wider flight position than the determined reference. The larger the settling time, the 

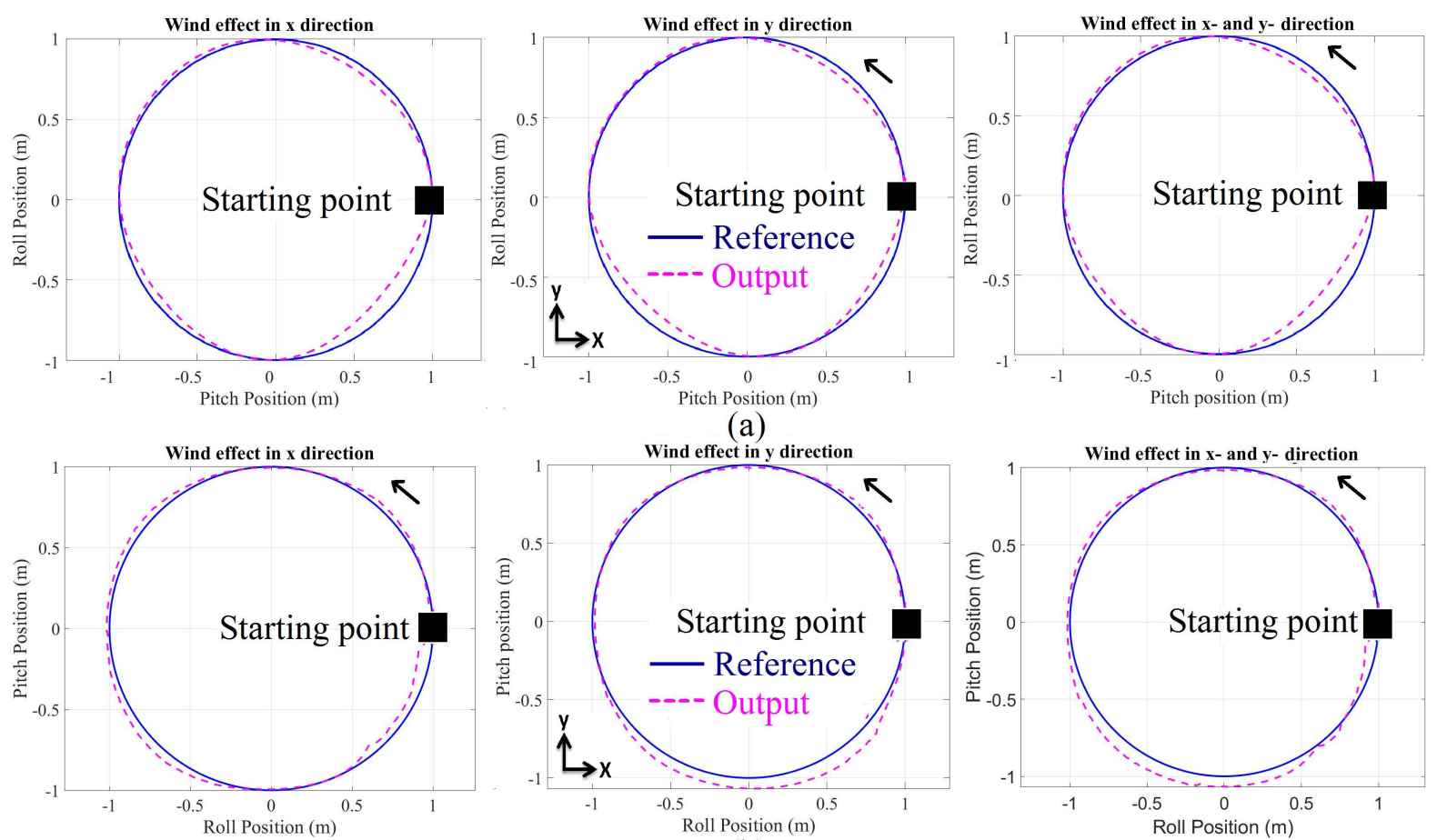

(b)

Figure 12: Results obtained with attitude control system with disturbance on each axis. (a) PID (b) Fuzzy-PID

greater the error of position shift will be. This principle applies for every combination of wind directions. Under wind effects in $\mathrm{x}$ - and $\mathrm{y}$ - direction, the position shift occurs accordingly and this result shows that the quadcopter can still maintain the position of the movement on circular trajectory. Details of position shift are as follows: maximum angle alteration of 0.17 radians and a position shift of $0.08 \mathrm{~m}$, occurred in the third and fourth quadrant. Due to the symmetrical shape of quadcopter between $\mathrm{X}$ - and $\mathrm{Y}$-axis, the angle alteration between roll and pitch must be linear so as to keep the position on a circular trajectory. When the control sistem exposed with disturbance parameter, which is the common occurence in real environments, the FuzzyPID is more reliable because it has the response system design through if-then logic parameters combined with adaptive PD control for error adjustment.

\section{Conclusions}

Quadcopter attitude movement modeling on a circular trajectory can be performed based on the Newton Euler kinematic model. A control system for continuous movement of the quadcopter is necessary to guarantee the quadcopter stability when maneuvering, where there are continuous change in pitch and roll angles. Without a control system, large amounts of overshoot were distinctly observable in the attitude movement in horizontal plane. To reduce the error, PID or Fuzzy-PID control can be added into the quadcopter movement modeling algorithm. In the case of the PID control system, only components proportional and derivative were needed to maintain quadcopter flying position stability. PD controller has maximum position shift of 0.12 $m$ on the circular trajectory, whereas in the case of Fuzzy-PID control system the shift is only $0.05 \mathrm{~m}$. This means that the quadcopter still moves on circular trajectory without significant error. Wind disturbances affect both control systems and significant error occurs in the third and 
fourth quadrant. Under wind disturbance of $2 \mathrm{~m} / \mathrm{s}$, the Fuzzy-PID control system can maintain the position on the circular trajectory better than the PID control system, with just $0.03 \mathrm{~m}$ of maximum position shift when compared to no disturbance. The Fuzzy-PID control system works as an adaptive PID controller, the tracking response is automatically adjusted based on the Fuzzy inference rules which allow comparing two inputs received by the Fuzzy control system so it can adapt to possible errors and avoid overshoot. Conclusively, the Fuzzy-PID controller is superior to the PID controller in which manual gain adjustment has a crucial role in quadcopter flight stability.

\section{Acknowledgment}

This work has been funded under the LEADERS - Erasmus Mundus Grant (agreement number 2014-0855/001-001) by European Commission, through the Education, Audiovisual and Culture Executive Agency, in the Action Plan 2 for the years 2014-2018, and supported by PNCDI III Programme P2 - Transfer of knowledge to the economic operator (Bridge Grant PN-III-P2 2.1 BG-2016-0296) funded by UEFISCDI, Romania.

\section{Bibliography}

[1] Ahmed S.F. et al (2015); Attitude Stabilization of Quad-rotor (UAV) System Using Fuzzy PID Controller, Second International Conference on Computing Technology and Information Management (ICCTIM), 99-104, 2015.

[2] Argentim L. M. et al (2013); PID, LQR and LQR-PID on a Quadcopter Platform, International conference on Informatics, Electronics and Vision (ICIEV), 1-6, 2013.

[3] Benic Z. et al (2016); Mathematical Modeling of Unmanned Aerial Vehicles with Four Rotors, Interdisciplinary Description of Complex System Journal, 14, 88-100, 2014.

[4] Bolandi H. et al (2013); Attitude Control of a Quadrotor with Optimized PID Controller, Intelligent Control and Automation Journal, 4(3), 335-342, 2013.

[5] Cai G. et al (2014); A Survey of Small-Scale Unmanned Aerial Vehicles: Recent Advances and Future Development Trends, Unmanned System Journal, 2, 1-25, 2014.

[6] Du Z.B., Lin T.C., Zhao T. B. (2015); Fuzzy Robust Tracking Control for Uncertain Nonlinear Time-Delay System, International Journal of Computers Communications $\&$ Control, 10(6):812-824, 2015.

[7] Fu C. et al (2013); UAS See-And-Avoid Strategy using a Fuzzy Logic Controller Optimized by Cross Entropy in Scaling Factors and Membership Functions, International Conference on Unmanned Aircraft System (ICUAS), 532-541, 2013.

[8] Gautam D., Ha C. (2013); Control of a Quadrotor Using a Smart Self-Tuning Fuzzy PID Controller, International Journal of Advanced Robotic Systems, 10:1-9, 2013.

[9] He Z., Zhao L. (2014); A Simple Attitude Control of Quadrotor Helicopter based on ZieglerNicols Rules for Tuning PD Parameters, The Scientific World Journal, 1-13, 2014.

[10] Kotarski D. et al (2016); Control Design for Unmanned Aerial Vehicle with Four Rotors, Interdisciplinary Description of Complex System Journal, 14(2), 236-245, 2016. 
[11] Lai J.G. et al. (2016); A New Adaptive Fuzzy PID Control Method and Its Application in FCBTM, International Journal of Computers Communications \& Control, ISSN 1841-9836, 11(3):394-404, 2016.

[12] Magnussen O. et al (2013); Experimental Validation of a Quaternion-based Attitude Estimation with Direct Input to A Quadcopter Control System, International Conference on Unmanned Aircraft System (ICUAS)l, 480-485, 2013.

[13] Mian A., Wang D. (2008); Modeling and Backstepping-based Nonlinear Control strategy for a 6 DOF Quadrotor Helicopter, Chinese Journal of Aeronauticsl, 21, 261-268, 2008.

[14] Moghaddam H. F., Vasegh N. (2014); Robust PID Stabilization of Linear Neutral TimeDelay System, International Journal of Computers Communications and Control, 9(2):201$208,2014$.

[15] Patel K., Barve J. (2014); Modeling, Simulation and Control Study for The Quadcopter UAV, The 9th International Conference on Industrial and Information Systems (ICIIS), $1-6,2014$.

[16] Sanchez E.N. et al. (2006); Combining Fuzzy, PID and Regulation Control for an autonomous mini helicopter, Information Sciences Journal, 177:1999-2022, 2006.

[17] Seidabad E.A. et al. (2014); Designing Fuzzy PID Controller for Quadrotor, International Journal of Advanced Research in Computer Science and Technology, 2:221-227, 2014.

[18] Stevanovic S. et al (2012); Robust Tracking Control Of A Quadrotor Helicopter Without Velocity Measurement, Annuals of DAAAM for 2012 and Proceedings of the 23rd International DAAAM Symposium, 595-600, 2012.

[19] Susnea I.; Vasiliu G. (2016); A Fuzzy Logic Software Tool and a New Scale for the Assessment of Creativity, International Journal of Computers Communications \& Control, ISSN 1841-9836, 11(3):441-449, 2016.

[20] Xu, H.; Vilanova, R. (2015); PI and Fuzzy Control for P-removal in Wastewater Treatment Plant, International Journal of Computers Communications \& Control, ISSN 1841-9836, 10(6):936-951, 2016.

[21] Yang, S. et al (2009); Design and Simulation of the Longitudinal Autopilot of UAV Based on Self-Adaptive Fuzzy PID Control, International Conference on Computational Intelligence and Security, 634-638, 2009.

[22] Ziegler J. G., Nichols N. B. (1942); Optimum Settings for Automatic Controllers, Transactions of the American Society of Mechanical Engineers, 64:759-768, 1942. 